\title{
A Quadruple Fixed Point Theorem for Contractive Type Condition by Using ICS Mapping and Application to Integral Equation
}

\author{
K.P.R. RaO, G.N.V. Kishore And K.V. Siva Parvathi
}

\begin{abstract}
In this paper, we obtain a Quadruple fixed point theorem for $\psi-\phi$ contractive condition in partially ordered partial metric spaces by using ICS mapping. We are also given an example and an application to integral equation which supports our main theorem.
\end{abstract}

\section{INTRODUCTION}

The notion of partial metric space was introduced by Matthews [8] as a part of the study of denotational semantics of data flow networks. In fact, it is widely recognized that partial metric spaces play an important role in constructing models in the theory of computation and domain theory in computer science.

First we recall some basic definitions and lemmas which play crucial role in the theory of partial metric spaces.

Definition 1.1. (See $[8,9]$ ) A partial metric on a nonempty set $X$ is a function $p: X \times X \rightarrow R^{+}$such that for all $x, y, z \in X$ :

$\left(p_{1}\right) x=y \Leftrightarrow p(x, x)=p(x, y)=p(y, y)$,

$\left(p_{2}\right) p(x, x) \leq p(x, y), p(y, y) \leq p(x, y)$,

$\left(p_{3}\right) p(x, y)=p(y, x)$,

$\left(p_{4}\right) p(x, y) \leq p(x, z)+p(z, y)-p(z, z)$.

The pair $(X, p)$ is called a partial metric space (PMS).

Clearly $p(x, y)=0$ implies $x=y$ and $x \neq y$ implies $p(x, y)>0$.

If $p$ is a partial metric on $X$, then the function $d_{p}: X \times X \rightarrow \mathbb{R}^{+}$given by $d_{p}(x, y)=2 p(x, y)-p(x, x)-p(y, y)$ is a metric on $X$.

Example 1.1 (See e.g. [9]). Consider $X=[0, \infty)$ with $p(x, y)=\max \{x, y\}$. Then $(X, p)$ is a partial metric space. It is clear that $p$ is not a (usual) metric. Note that in this case $d_{p}(x, y)=|x-y|$.

2010 Mathematics Subject Classification. Primary: 47H10, 54H25.

Key words and phrases. Partial metric space, Quadruple fixed point, ICS mapping, mixed monotone property. 
Each partial metric $p$ on $X$ generates a $T_{0}$ topology $\tau_{p}$ on $X$ which has as a base the family of open $p$-balls $\left\{B_{p}(x, \varepsilon), x \in X, \varepsilon>0\right\}$, where $B_{p}(x, \varepsilon)=$ $\{y \in X: p(x, y)<p(x, x)+\varepsilon\}$ for all $x \in X$ and $\varepsilon>0$.

We now state some basic topological notions (such as convergence, completeness) on partial metric spaces (see e.g. $[1,2,8,9]$.)

\section{Definition 1.2.}

(1) A sequence $\left\{x_{n}\right\}$ in the PMS $(X, p)$ converges to the limit $x$ if and only if $p(x, x)=\lim _{n \rightarrow \infty} p\left(x, x_{n}\right)$.

(2) A sequence $\left\{x_{n}\right\}$ in the PMS $(X, p)$ is called a Cauchy sequence if $\lim _{n, m \rightarrow \infty} p\left(x_{n}, x_{m}\right)$ exists and is finite.

(3) A PMS $(X, p)$ is called complete if every Cauchy sequence $\left\{x_{n}\right\}$ in $X$ converges with respect to $\tau_{p}$, to a point $x \in X$ such that $p(x, x)=\lim _{n, m \rightarrow \infty} p\left(x_{n}, x_{m}\right)$.

We need the following lemmas in $\operatorname{PMS}([8,9])$.

\section{Lemma 1.1.}

(1) A sequence $\left\{x_{n}\right\}$ is a Cauchy sequence in the PMS $(X, p)$ if and only if it is a Cauchy sequence in the metric space $\left(X, d_{p}\right)$.

(2) A PMS $(X, p)$ is complete if and only if the metric space $\left(X, d_{p}\right)$ is complete. Moreover

$$
\lim _{n \rightarrow \infty} d_{p}\left(x, x_{n}\right)=0 \Leftrightarrow p(x, x)=\lim _{n \rightarrow \infty} p\left(x, x_{n}\right)=\lim _{n, m \rightarrow \infty} p\left(x_{n}, x_{m}\right) .
$$

Lemma 1.2. Assume $x_{n} \rightarrow z$ as $n \rightarrow \infty$ in a $P M S(X, p)$ such that $p(z, z)=$ 0 . Then $\lim _{n \rightarrow \infty} p\left(x_{n}, y\right)=p(z, y)$ for every $y \in X$.

In 2009, K.P. Chi [3] introduced the concept of ICS mapping as follows.

Definition $1.3([3,7])$. Let $(X, d)$ be a metric space. A mapping $T: X \rightarrow X$ is said to be ICS if $T$ is injective, continuous and has the property: for every sequence $\left\{x_{n}\right\}$ in $X$, if $\left\{T x_{n}\right\}$ is convergent then $\left\{x_{n}\right\}$ is also convergent.

Now we introduce the notion of Quadruple fixed point as follows.

Definition 1.4. An element $(x, y, z, w) \in X^{4}$ is called a Quadruple fixed point of $F: X^{4} \rightarrow X$ if $F(x, y, z, w)=x, F(y, z, w, x)=y, F(z, w, x, y)=z$ and $F(w, x, y, z)=w$.

Definition $1.5([5,6])$. Let $(X, \preceq)$ be a partial ordered set and $F: X^{4} \rightarrow X$. We say that $F$ has the mixed monotone property if $F(x, y, z, w)$ is monotone non decreasing in $x$ and $z$, and is monotone non - increasing in $y$ and $w$, 
that is, for any $x, y, z, w \in X$.

$$
\begin{aligned}
& x_{1}, x_{2} \in X, x_{1} \preceq x_{2} \Rightarrow \quad F\left(x_{1}, y, z, w\right) \preceq F\left(x_{2}, y, z, w\right), \\
& y_{1}, y_{2} \in X, y_{1} \preceq y_{2} \Rightarrow \quad F\left(x, y_{1}, z, w\right) \succeq F\left(x, y_{2}, z, w\right), \\
& z_{1}, z_{2} \in X, z_{1} \preceq z_{2} \quad \Rightarrow \quad F\left(x, y, z_{1}, w\right) \preceq F\left(x, y, z_{2}, w\right), \\
& w_{1}, w_{2} \in X, w_{1} \preceq w_{2} \quad \Rightarrow \quad F\left(x, y, z, w_{1}\right) \succeq F\left(x, y, z, w_{2}\right) .
\end{aligned}
$$

\section{Main Results}

Let $\Psi$ denote the set of all continuous and monotonically increasing functions $\psi:[0, \infty) \rightarrow[0, \infty)$ with $\psi(0)=0$.

Let $\Phi$ denote the set of all lower semi continuous functions $\phi:[0, \infty) \rightarrow$ $[0, \infty)$ such that $\phi(t)>0$ for $t>0$ and $\phi(0)=0$.

Let $(X, \preceq)$ be a partial ordered set. We consider the following partial order on the product space $X^{4}=X \times X \times X \times X$. $(x, y, z, w) \preceq(u, v, r, t)$ iff $x \preceq u, y \succeq v, z \preceq r$ and $w \succeq t$ where $(x, y, z, w)$ and $(u, v, r, t) \in X^{4}$.

Theorem 2.1. Let $(X, p, \preceq)$ be a complete ordered partial metric space. Suppose $T: X \rightarrow X$ is an ICS mapping and $F: X^{4} \rightarrow X$ is such that $F$ has the mixed monotone property. Assume that there exist $\psi \in \Psi$ and $\phi \in \Phi$ such that

$$
\begin{aligned}
& \psi(p(T F(x, y, z, w), T F(u, v, r, t))) \\
& \leq \psi\left(\max \left\{\begin{array}{c}
p(T x, T u), p(T y, T v), \\
p(T z, T r), p(T w, T t)
\end{array}\right\}\right) \\
& -\phi\left(\max \left\{\begin{array}{c}
p(T x, T u), p(T y, T v), \\
p(T z, T r), p(T w, T t)
\end{array}\right\}\right),
\end{aligned}
$$

for all $x, y, z, w, u, v, r, t \in X$ for which $x \preceq u, y \succeq v, z \preceq r$ and $w \succeq t$.

Suppose $X$ has the following property $(A)$ :

I. If non - decreasing sequence $x_{n} \rightarrow x$, then $x_{n} \preceq x$ for all $n$,

II. if non - increasing sequence $y_{n} \rightarrow y$, then $y_{n} \succeq y$ for all $n$.

Suppose there exist $x_{0}, y_{0}, z_{0}, w_{0} \in X$ such that

$$
\begin{aligned}
& x_{0} \preceq F\left(x_{0}, y_{0}, z_{0}, w_{0}\right), \\
& y_{0} \succeq F\left(y_{0}, z_{0}, w_{0}, x_{0}\right), \\
& z_{0} \preceq F\left(z_{0}, w_{0}, x_{0}, y_{0}\right), \\
& w_{0} \succeq F\left(w_{0}, x_{0}, y_{0}, z_{0}\right) .
\end{aligned}
$$

Then there exist $x, y, z, w \in X$ such that

$$
\begin{aligned}
& F(x, y, z, w)=x, \quad F(y, z, w, x)=y, \\
& F(z, w, x, y)=z, \quad F(w, x, y, z)=w,
\end{aligned}
$$

that is, $F$ has a quadruple fixed point. 
Proof. Let $x_{0}, y_{0}, z_{0}, w_{0} \in X$ be such that

$$
\begin{aligned}
x_{0} & \preceq F\left(x_{0}, y_{0}, z_{0}, w_{0}\right), \\
y_{0} & \succeq F\left(y_{0}, z_{0}, w_{0}, x_{0}\right), \\
z_{0} & \preceq F\left(z_{0}, w_{0}, x_{0}, y_{0}\right), \\
w_{0} & \succeq F\left(w_{0}, x_{0}, y_{0}, z_{0}\right) .
\end{aligned}
$$

Set

$$
\begin{aligned}
x_{1} & =F\left(x_{0}, y_{0}, z_{0}, w_{0}\right) \succeq x_{0}, \\
y_{1} & =F\left(y_{0}, z_{0}, w_{0}, x_{0}\right) \preceq y_{0}, \\
z_{1} & =F\left(z_{0}, w_{0}, x_{0}, y_{0}\right) \succeq z o, \\
w_{1} & =F\left(w_{0}, x_{0}, y_{0}, z_{0}\right) \preceq w_{0}
\end{aligned}
$$

and by the mixed monotone property of $F$, for $n \geq 1$, inductively we get

$$
\begin{aligned}
x_{n} & =F\left(x_{n-1}, y_{n-1}, z_{n-1}, w_{n-1}\right) \succeq x_{n-1} \succeq \cdots \succeq x_{0}, \\
y_{n} & =F\left(y_{n-1}, z_{n-1}, w_{n-1}, x_{n-1}\right) \preceq y_{n-1} \preceq \cdots \preceq y_{0}, \\
z_{n} & =F\left(z_{n-1}, w_{n-1}, x_{n-1}, y_{n-1}\right) \succeq z_{n-1} \succeq \cdots \succeq z_{0}, \\
w_{n} & =F\left(w_{n-1}, x_{n-1}, y_{n-1}, z_{n-1}\right) \preceq w_{n-1} \preceq \cdots \preceq w_{0} .
\end{aligned}
$$

Assume for some $n \in N, x_{n+1}=x_{n}, y_{n+1}=y_{n}, z_{n+1}=z_{n}$ and $w_{n+1}=$ $w_{n}$.

Then $\left(x_{n}, y_{n}, z_{n}, w_{n}\right)$ is a quadruple fixed point of $F$. Hence the theorem.

Now assume that $x_{n+1} \neq x_{n}$ or $y_{n+1} \neq y_{n}$ or $z_{n+1} \neq z_{n}$ or $w_{n+1} \neq w_{n}$ for any $n \in N$.

Since $T$ is injective, we have

$$
\begin{aligned}
a_{n}=\max \left\{p \left(T x_{n+1}\right.\right. & \left.\left., T x_{n}\right), p\left(T y_{n+1}, T y_{n}\right), p\left(T z_{n+1}, T z_{n}\right), p\left(T w_{n+1}, T w_{n}\right)\right\}>0 \\
\psi\left(p\left(T x_{n+1}, T x_{n}\right)\right)= & \psi\left(p\left(T F\left(x_{n}, y_{n}, z_{n}, w_{n}\right), T F\left(x_{n-1}, y_{n-1}, z_{n-1}, w_{n-1}\right)\right)\right) \\
\leq & \psi\left(\max \left\{\begin{array}{l}
p\left(T x_{n}, T x_{n-1}\right), p\left(T y_{n}, T y_{n-1}\right), \\
p\left(T z_{n}, T z_{n-1}\right), p\left(T w_{n}, T w_{n-1}\right)
\end{array}\right\}\right) \\
& -\phi\left(\max \left\{\begin{array}{l}
p\left(T x_{n}, T x_{n-1}\right), p\left(T y_{n}, T y_{n-1}\right), \\
p\left(T z_{n}, T z_{n-1}\right), p\left(T w_{n}, T w_{n-1}\right)
\end{array}\right\}\right) \\
= & \psi\left(a_{n-1}\right)-\phi\left(a_{n-1}\right) .
\end{aligned}
$$

Similarly,

$$
\begin{aligned}
\psi\left(p\left(T y_{n+1}, T y_{n}\right)\right) & \leq \psi\left(a_{n-1}\right)-\phi\left(a_{n-1}\right), \\
\psi\left(p\left(T z_{n+1}, T z_{n}\right)\right) & \leq \psi\left(a_{n-1}\right)-\phi\left(a_{n-1}\right), \\
\psi\left(p\left(T w_{n+1}, T w_{n}\right)\right) & \leq \psi\left(a_{n-1}\right)-\phi\left(a_{n-1}\right) .
\end{aligned}
$$


Hence

$$
\begin{aligned}
\psi\left(a_{n}\right) & =\psi\left(\max \left\{\begin{array}{l}
p\left(T x_{n+1}, T x_{n}\right), p\left(T y_{n+1}, T y_{n}\right), \\
p\left(T z_{n+1}, T z_{n}\right), p\left(T w_{n+1}, T w_{n}\right)
\end{array}\right\}\right) \\
& =\max \left\{\begin{array}{c}
\psi\left(p\left(T x_{n+1}, T x_{n}\right)\right), \psi\left(p\left(T y_{n+1}, T y_{n}\right)\right), \\
\psi\left(p\left(T z_{n+1}, T z_{n}\right)\right), \psi\left(p\left(T w_{n+1}, T w_{n}\right)\right)
\end{array}\right\} \\
& \leq \psi\left(a_{n-1}\right)-\phi\left(a_{n-1}\right) .
\end{aligned}
$$

Thus

$$
\begin{aligned}
\psi\left(a_{n}\right) & \leq \psi\left(a_{n-1}\right)-\phi\left(a_{n-1}\right) \\
& <\psi\left(a_{n-1}\right) .
\end{aligned}
$$

Since $\psi$ is increasing, we have

$$
a_{n}<a_{n-1}, \forall n=1,2,3, \cdots
$$

Thus $\left\{a_{n}\right\}$ is a positive decreasing sequence of real numbers. Hence there exists $r \geq 0$ such that $\lim _{n \rightarrow \infty} a_{n}=r$. Suppose $r>0$.

Letting $n \rightarrow \infty$ in (1), we obtain that

$$
\psi(r) \leq \psi(r)-\phi(r)<\psi(r) .
$$

a contradiction. Hence $r=0$.

Thus

$$
\lim _{n \rightarrow \infty} \max \left\{\begin{array}{l}
p\left(T x_{n+1}, T x_{n}\right), p\left(T y_{n+1}, T y_{n}\right), \\
p\left(T z_{n+1}, T z_{n}\right), p\left(T w_{n+1}, T w_{n}\right)
\end{array}\right\}=0 .
$$

From $\left(p_{2}\right)$, we have

(3) $\lim _{n \rightarrow \infty} \max \left\{p\left(T x_{n}, T x_{n}\right), p\left(T y_{n}, T y_{n}\right), p\left(T z_{n}, T z_{n}\right), p\left(T w_{n}, T w_{n}\right)\right\}=0$.

From definition of $d_{p}$ and from (2) and (3), it follows that

$$
\lim _{n \rightarrow \infty} \max \left\{\begin{array}{l}
d_{p}\left(T x_{n+1}, T x_{n}\right), d_{p}\left(T y_{n+1}, T y_{n}\right), \\
d_{p}\left(T z_{n+1}, T z_{n}\right), d_{p}\left(T w_{n+1}, T w_{n}\right)
\end{array}\right\}=0 .
$$

Now we shall prove that $\left\{T x_{n}\right\},\left\{T y_{n}\right\},\left\{T z_{n}\right\}$ and $\left\{T w_{n}\right\}$ are Cauchy sequences in the metric space $\left(X, d_{p}\right)$. Assume on the contrary that $\left\{T x_{n}\right\}$ or $\left\{T y_{n}\right\}$ or $\left\{T z_{n}\right\}$ or $\left\{T w_{n}\right\}$ is not a Cauchy sequence in $\left(X, d_{p}\right)$.

Then there exists $\epsilon>0$ for which we can find subsequences of integers $\left\{m_{k}\right\}$ and $\left\{n_{k}\right\}$ with $n_{k}>m_{k}>k$ such that

$$
\max \left\{\begin{array}{l}
d_{p}\left(T x_{m_{k}}, T x_{n_{k}}\right), d_{p}\left(T y_{m_{k}}, T y_{n_{k}}\right), \\
d_{p}\left(T z_{m_{k}}, T z_{n_{k}}\right), d_{p}\left(T w_{m_{k}}, T w_{n_{k}}\right)
\end{array}\right\} \geq \epsilon
$$

Further, corresponding to $m_{k}$, we may choose $n_{k}$ such that it is the smallest integer satisfying (5) and $n_{k}>m_{k}$. Then

$$
\max \left\{\begin{array}{l}
d_{p}\left(T x_{m_{k}}, T x_{n_{k}-1}\right), d_{p}\left(T y_{m_{k}}, T y_{n_{k}-1}\right), \\
d_{p}\left(T z_{m_{k}}, T z_{n_{k}-1}\right), d_{p}\left(T w_{m_{k}}, T w_{n_{k}-1}\right)
\end{array}\right\}<\epsilon .
$$


We have

$$
\begin{gathered}
d_{p}\left(T x_{m_{k}}, T x_{n_{k}}\right) \leq d_{p}\left(T x_{m_{k}}, T x_{m_{k}-1}\right) \\
\quad+d_{p}\left(T x_{m_{k}-1}, T x_{n_{k}-1}\right)+d_{p}\left(T x_{n_{k}-1}, T x_{n_{k}}\right) \\
\leq d_{p}\left(T x_{m_{k}}, T x_{m_{k}-1}\right)+d_{p}\left(T x_{m_{k}-1}, T x_{m_{k}}\right) \\
d_{p}\left(T x_{m_{k}}, T x_{n_{k}-1}\right)+d_{p}\left(T x_{n_{k}-1}, T x_{n_{k}}\right) \\
<2 d_{p}\left(T x_{m_{k}}, T x_{m_{k}-1}\right)+\epsilon+d_{p}\left(T x_{n_{k}-1}, T x_{n_{k}}\right) \text {, from (6). }
\end{gathered}
$$

Letting $k \rightarrow \infty$ in (7) and (8) and using (4), we get

$$
\lim _{k \rightarrow \infty} d_{p}\left(T x_{m_{k}}, T x_{n_{k}}\right) \leq \lim _{k \rightarrow \infty} d_{p}\left(T x_{m_{k}-1}, T x_{n_{k}-1}\right) \leq \epsilon .
$$

Similarly,

$$
\begin{aligned}
& \lim _{k \rightarrow \infty} d_{p}\left(T y_{m_{k}}, T y_{n_{k}}\right) \leq \lim _{k \rightarrow \infty} d_{p}\left(T y_{m_{k}-1}, T y_{n_{k}-1}\right) \leq \epsilon, \\
& \lim _{k \rightarrow \infty} d_{p}\left(T z_{m_{k}}, T z_{n_{k}}\right) \leq \lim _{k \rightarrow \infty} d_{p}\left(T z_{m_{k}-1}, T z_{n_{k}-1}\right) \leq \epsilon, \\
& \lim _{k \rightarrow \infty} d_{p}\left(T w_{m_{k}}, T w_{n_{k}}\right) \leq \lim _{k \rightarrow \infty} d_{p}\left(T w_{m_{k}-1}, T w_{n_{k}-1}\right) \leq \epsilon,
\end{aligned}
$$

Using (5) and (9) - (12), we have

$$
\begin{aligned}
\epsilon & \leq \lim _{k \rightarrow \infty} \max \left\{\begin{array}{l}
d_{p}\left(T x_{m_{k}}, T x_{n_{k}}\right), d_{p}\left(T y_{m_{k}}, T y_{n_{k}}\right), \\
d_{p}\left(T z_{m_{k}}, T z_{n_{k}}\right), d_{p}\left(T w_{m_{k}}, T w_{n_{k}}\right)
\end{array}\right\} \\
& \leq \lim _{k \rightarrow \infty} \max \left\{\begin{array}{l}
d_{p}\left(T x_{m_{k}-1}, T x_{n_{k}-1}\right), d_{p}\left(T y_{m_{k}-1}, T y_{n_{k}-1}\right), \\
d_{p}\left(T z_{m_{k}-1}, T z_{n_{k}-1}\right), d_{p}\left(T w_{m_{k}-1}, T w_{n_{k}-1}\right)
\end{array}\right\} \\
& \leq \epsilon
\end{aligned}
$$

Now using (13) and (3), we obtain

$$
\epsilon=\lim _{k \rightarrow \infty} \max \left\{\begin{array}{c}
2 p\left(T x_{m_{k}}, T x_{n_{k}}\right)-p\left(T x_{m_{k}}, T x_{m_{k}}\right)-p\left(T x_{n_{k}}, T x_{n_{k}}\right) \\
2 p\left(T y_{m_{k}}, T y_{n_{k}}\right)-p\left(T y_{m_{k}}, T y_{m_{k}}\right)-p\left(T y_{n_{k}}, T y_{n_{k}}\right) \\
2 p\left(T z_{m_{k}}, T z_{n_{k}}\right)-p\left(T z_{m_{k}}, T z_{m_{k}}\right)-p\left(T z_{n_{k}}, T z_{n_{k}}\right) \\
2 p\left(T w_{m_{k}}, T w_{n_{k}}\right)-p\left(T w_{m_{k}}, T w_{m_{k}}\right)-p\left(T w_{n_{k}}, T w_{n_{k}}\right)
\end{array}\right\},
$$

$$
\frac{\epsilon}{2}=\lim _{k \rightarrow \infty} \max \left\{\begin{array}{l}
p\left(T x_{m_{k}}, T x_{n_{k}}\right), p\left(T y_{m_{k}}, T y_{n_{k}}\right) \\
p\left(T z_{m_{k}}, T z_{n_{k}}\right), p\left(T w_{m_{k}}, T w_{n_{k}}\right)
\end{array}\right\}
$$

Similarly from (13) and (14), we obtain

$$
\frac{\epsilon}{2}=\lim _{k \rightarrow \infty} \max \left\{\begin{array}{l}
p\left(T x_{m_{k}-1}, T x_{n_{k}-1}\right), p\left(T y_{m_{k}-1}, T y_{n_{k}-1}\right) \\
p\left(T z_{m_{k}-1}, T z_{n_{k}-1}\right), p\left(T w_{m_{k}-1}, T w_{n_{k}-1}\right)
\end{array}\right\} .
$$


Now using (2.1.1), we have

$$
\begin{aligned}
& \psi\left(p\left(T x_{m_{k}}, T x_{n_{k}}\right)\right) \\
&= \psi\left(p\left(F\left(x_{m_{k}-1}, y_{m_{k}-1}, z_{m_{k}-1}, w_{m_{k}-1}\right), F\left(x_{n_{k}-1}, y_{n_{k}-1}, z_{n_{k}-1}, w_{n_{k}-1}\right)\right)\right) \\
& \leq \psi\left(\max \left\{\begin{array}{l}
p\left(T x_{m_{k}-1}, T x_{n_{k}-1}\right), p\left(T y_{m_{k}-1}, T y_{n_{k}-1}\right), \\
p\left(T z_{m_{k}-1}, T z_{n_{k}-1}\right), p\left(T w_{m_{k}-1}, T w_{n_{k}-1}\right)
\end{array}\right\}\right) \\
& \quad-\phi\left(\max \left\{\begin{array}{c}
p\left(T x_{m_{k}-1}, T x_{n_{k}-1}\right), p\left(T y_{m_{k}-1}, T y_{n_{k}-1}\right), \\
p\left(T z_{m_{k}-1}, T z_{n_{k}-1}\right), p\left(T w_{m_{k}-1}, T w_{n_{k}-1}\right)
\end{array}\right\}\right) .
\end{aligned}
$$

Similarly,

$$
\begin{aligned}
& \psi\left(p\left(T y_{m_{k}}, T y_{n_{k}}\right)\right) \\
& \leq \psi\left(\max \left\{\begin{array}{c}
p\left(T x_{m_{k}-1}, T x_{n_{k}-1}\right), p\left(T y_{m_{k}-1}, T y_{n_{k}-1}\right), \\
p\left(T z_{m_{k}-1}, T z_{n_{k}-1}\right), p\left(T w_{m_{k}-1}, T w_{n_{k}-1}\right)
\end{array}\right\}\right) \\
& \quad-\phi\left(\max \left\{\begin{array}{c}
p\left(T x_{m_{k}-1}, T x_{n_{k}-1}\right), p\left(T y_{m_{k}-1}, T y_{n_{k}-1}\right), \\
p\left(T z_{m_{k}-1}, T z_{n_{k}-1}\right), p\left(T w_{m_{k}-1}, T w_{n_{k}-1}\right)
\end{array}\right\}\right), \\
& \psi\left(p\left(T z_{m_{k}}, T z_{n_{k}}\right)\right) \\
& \leq \psi\left(\max \left\{\begin{array}{l}
p\left(T x_{m_{k}-1}, T x_{n_{k}-1}\right), p\left(T y_{m_{k}-1}, T y_{n_{k}-1}\right), \\
p\left(T z_{m_{k}-1}, T z_{n_{k}-1}\right), p\left(T w_{m_{k}-1}, T w_{n_{k}-1}\right)
\end{array}\right\}\right) \\
& \quad-\phi\left(\max \left\{\begin{array}{l}
p\left(T x_{m_{k}-1}, T x_{n_{k}-1}\right), p\left(T y_{m_{k}-1}, T y_{n_{k}-1}\right), \\
p\left(T z_{m_{k}-1}, T z_{n_{k}-1}\right), p\left(T w_{m_{k}-1}, T w_{n_{k}-1}\right)
\end{array}\right\}\right)
\end{aligned}
$$

and

$$
\begin{aligned}
& \psi\left(p\left(T w_{m_{k}}, T w_{n_{k}}\right)\right) \\
& \leq \psi\left(\max \left\{\begin{array}{l}
p\left(T x_{m_{k}-1}, T x_{n_{k}-1}\right), p\left(T y_{m_{k}-1}, T y_{n_{k}-1}\right), \\
p\left(T z_{m_{k}-1}, T z_{n_{k}-1}\right), p\left(T w_{m_{k}-1}, T w_{n_{k}-1}\right)
\end{array}\right\}\right) \\
& \quad-\phi\left(\max \left\{\begin{array}{l}
p\left(T x_{m_{k}-1}, T x_{n_{k}-1}\right), p\left(T y_{m_{k}-1}, T y_{n_{k}-1}\right), \\
p\left(T z_{m_{k}-1}, T z_{n_{k}-1}\right), p\left(T w_{m_{k}-1}, T w_{n_{k}-1}\right)
\end{array}\right\}\right) .
\end{aligned}
$$

Thus

$$
\begin{aligned}
& \psi(\max \{ p\left(T x_{m_{k}}, T x_{n_{k}}\right), p\left(T y_{m_{k}}, T y_{n_{k}}\right), \\
&\left.\left.p\left(T z_{m_{k}}, T z_{n_{k}}\right), p\left(T w_{m_{k}}, T w_{n_{k}}\right)\right\}\right) \\
&=\max \left\{\psi\left(p\left(T x_{m_{k}}, T x_{n_{k}}\right)\right), \psi\left(p\left(T y_{m_{k}}, T y_{n_{k}}\right)\right),\right.\left.\psi\left(p\left(T z_{m_{k}}, T z_{n_{k}}\right)\right), \psi\left(p\left(T w_{m_{k}}, T w_{n_{k}}\right)\right)\right\} \\
& \leq \psi\left(\max \left\{\begin{array}{l}
p\left(T x_{m_{k}-1}, T x_{n_{k}-1}\right), p\left(T y_{m_{k}-1}, T y_{n_{k}-1}\right), \\
p\left(T z_{m_{k}-1}, T z_{n_{k}-1}\right), p\left(T w_{m_{k}-1}, T w_{n_{k}-1}\right)
\end{array}\right\}\right) \\
&-\phi\left(\max \left\{\begin{array}{l}
p\left(T x_{m_{k}-1}, T x_{n_{k}-1}\right), p\left(T y_{m_{k}-1}, T y_{n_{k}-1}\right), \\
p\left(T z_{m_{k}-1}, T z_{n_{k}-1}\right), p\left(T w_{m_{k}-1}, T w_{n_{k}-1}\right)
\end{array}\right\}\right) .
\end{aligned}
$$


Letting $k \rightarrow \infty$ and using (14) and (15), we obtain

$$
\psi\left(\frac{\epsilon}{2}\right) \leq \psi\left(\frac{\epsilon}{2}\right)-\phi\left(\frac{\epsilon}{2}\right)<\psi\left(\frac{\epsilon}{2}\right)
$$

a contradiction.

Thus $\left\{T x_{n}\right\},\left\{T y_{n}\right\},\left\{T z_{n}\right\}$ and $\left\{T w_{n}\right\}$ are Cauchy sequences in the metric space $\left(X, d_{p}\right)$. That is

$$
\begin{array}{ll}
\lim _{m, n \rightarrow \infty} d_{p}\left(T x_{n}, T x_{m}\right) & =0, \\
\lim _{m, n \rightarrow \infty} d_{p}\left(T z_{n}, T z_{m}\right)=0 & d_{p}\left(T y_{n}, T y_{m}\right)=0, \\
\lim _{m, n \rightarrow \infty} d_{p}\left(T w_{n}, T w_{m}\right) & =0 .
\end{array}
$$

From the definition of $d_{p}$ and from (3), we have

$$
\left.\begin{array}{rlrl}
\lim _{m, n \rightarrow \infty} p\left(T x_{n}, T x_{m}\right) & =0, & \lim _{m, n \rightarrow \infty} p\left(T y_{n}, T y_{m}\right) & =0, \\
\lim _{m, n \rightarrow \infty} p\left(T z_{n}, T z_{m}\right) & =0, & \lim _{m, n \rightarrow \infty} p\left(T w_{n}, T w_{m}\right) & =0 .
\end{array}\right\}
$$

Thus $\left\{T x_{n}\right\},\left\{T y_{n}\right\},\left\{T z_{n}\right\}$ and $\left\{T w_{n}\right\}$ are Cauchy sequences in $(X, p)$.

Since $(X, p)$ is complete, the Cauchy sequences $\left\{T x_{n}\right\},\left\{T y_{n}\right\},\left\{T z_{n}\right\}$ and $\left\{T w_{n}\right\}$ are convergent.

Since $T$ is an ICS mapping, there exist $x, y, z, w \in X$ such that

$$
\begin{aligned}
\lim _{n \rightarrow \infty} p\left(x_{n}, x\right)=p(x, x), & \lim _{n \rightarrow \infty} p\left(y_{n}, y\right)=p(y, y), \\
\lim _{n \rightarrow \infty} p\left(z_{n}, z\right)=p(z, z), & \lim _{n \rightarrow \infty} p\left(w_{n}, w\right)=p(w, w) .
\end{aligned}
$$

Since $T$ is continuous, we have

$$
\begin{array}{rlrl}
\lim _{n \rightarrow \infty} p\left(T x_{n}, T x\right) & =p(T x, T x), & & \lim _{n \rightarrow \infty} p\left(T y_{n}, T y\right)=p(T y, T y), \\
\lim _{n \rightarrow \infty} p\left(T z_{n}, T z\right)=p(T z, T z), & \lim _{n \rightarrow \infty} p\left(T w_{n}, T w\right)=p(T w, T w) .
\end{array}
$$

These implies that $\left\{T x_{n}\right\},\left\{T y_{n}\right\},\left\{T z_{n}\right\}$ and $\left\{T w_{n}\right\}$ are convergent to $T x$, $T y, T z$ and $T w$ respectively. Using Lemma 1.1 (2) and from (16), it follows that

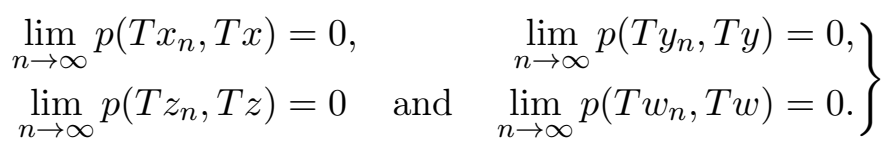

Suppose $X$ has the property $(A)$.

Since $\left\{x_{n}\right\},\left\{z_{n}\right\}$ are non - decreasing with $x_{n} \rightarrow x, z_{n} \rightarrow z$ and also $\left\{y_{n}\right\},\left\{w_{n}\right\}$ are non - increasing with $y_{n} \rightarrow y, w_{n} \rightarrow w$ then by the property $(A)$, we have $x_{n} \preceq x, y_{n} \succeq y, z_{n} \preceq z$ and $w_{n} \succeq w$ for all $n$. 
Consider now

$$
\begin{aligned}
\psi\left(p\left(T x_{n+1}, T F(x, y, z, w)\right)\right)= & \psi\left(p\left(T F\left(x_{n}, y_{n}, z_{n}, w_{n}\right), T F(x, y, z, w)\right)\right) \\
\leq \psi & \left(\max \left\{\begin{array}{c}
p\left(T x_{n}, T x\right), p\left(T y_{n}, T y\right) \\
p\left(T z_{n}, T z\right), p\left(T w_{n}, T w\right)
\end{array}\right\}\right) \\
& -\phi\left(\max \left\{\begin{array}{c}
p\left(T x_{n}, T x\right), p\left(T y_{n}, T y\right) \\
p\left(T z_{n}, T z\right), p\left(T w_{n}, T w\right)
\end{array}\right\}\right) .
\end{aligned}
$$

Taking $n \rightarrow \infty$ and using (17), we get

$$
p(T x, T F(x, y, z, w))=0
$$

so that $T x=T F(x, y, z, w)$. Since $T$ is injective, we obtain $x=F(x, y, z, w)$.

Similarly we can show that $y=F(y, z, w, x), z=F(z, w, x, y), w=$ $F(w, x, y, z)$.

Thus $(x, y, z, w)$ is a quadruple fixed point of $F$.

Theorem 2.2. Let $(X, p)$ be a complete partial metric space. Suppose $T$ : $X \rightarrow X$ is an ICS mapping and $F: X^{4} \rightarrow X$. Assume that there exist $\psi \in \Psi$ and $\phi \in \Phi$ such that

$$
\begin{aligned}
& \psi(p(T F(x, y, z, w), T F(u, v, r, t))) \\
& \leq \psi\left(\max \left\{\begin{array}{c}
p(T x, T u), p(T y, T v), \\
p(T z, T r), p(T w, T t)
\end{array}\right\}\right) \\
& \quad-\phi\left(\max \left\{\begin{array}{c}
p(T x, T u), p(T y, T v), \\
p(T z, T r), p(T w, T t)
\end{array}\right\}\right),
\end{aligned}
$$

for all $x, y, z, w, u, v, r, t \in X$.

Then $F$ has a quadruple fixed point of the form $(x, x, x, x)$ where $x \in X$.

Proof. By proceeding the proof of Theorem 2.1, we get

$$
\begin{aligned}
& F(x, y, z, w)=x, \quad F(y, z, w, x)=y \\
& F(z, w, x, y)=z, \quad F(w, x, y, z)=w
\end{aligned}
$$

for some $x, y, z, w \in X$.

Now from (2.2.1), we have

$$
\begin{aligned}
\psi(p(T x, T y))=\psi & (p(T F(x, y, z, w), T F(y, z, w, x))) \\
\leq \psi & \left(\max \left\{\begin{array}{l}
p(T x, T y), p(T y, T z), \\
p(T z, T w), p(T w, T x)
\end{array}\right\}\right) \\
& -\phi\left(\max \left\{\begin{array}{c}
p(T x, T y), p(T y, T z), \\
p(T z, T w), p(T w, T x)
\end{array}\right\}\right),
\end{aligned}
$$


Similarly, we have

$$
\begin{aligned}
\psi(p(T y, T z)) \leq \psi & \left(\max \left\{\begin{array}{l}
p(T x, T y), p(T y, T z), \\
p(T z, T w), p(T w, T x)
\end{array}\right\}\right) \\
& -\phi\left(\max \left\{\begin{array}{l}
p(T x, T y), p(T y, T z), \\
p(T z, T w), p(T w, T x)
\end{array}\right\}\right), \\
\psi(p(T z, T w)) \leq \psi & \left(\max \left\{\begin{array}{l}
p(T x, T y), p(T y, T z), \\
p(T z, T w), p(T w, T x)
\end{array}\right\}\right) \\
& -\phi\left(\max \left\{\begin{array}{l}
p(T x, T y), p(T y, T z), \\
p(T z, T w), p(T w, T x)
\end{array}\right\}\right),
\end{aligned}
$$

and

$$
\begin{aligned}
\psi(p(T w, T x)) \leq \psi & \left(\max \left\{\begin{array}{l}
p(T x, T y), p(T y, T z), \\
p(T z, T w), p(T w, T x)
\end{array}\right\}\right) \\
& -\phi\left(\max \left\{\begin{array}{l}
p(T x, T y), p(T y, T z), \\
p(T z, T w), p(T w, T x)
\end{array}\right\}\right),
\end{aligned}
$$

Now

$$
\begin{gathered}
\psi\left(\max \left\{\begin{array}{l}
p(T x, T y), p(T y, T z), \\
p(T z, T w), p(T w, T x)
\end{array}\right\}\right) \\
=\max \left\{\begin{array}{l}
\psi(p(T x, T y)), \psi(p(T y, T z)), \\
\psi(p(T z, T w)), \psi(p(T w, T x))
\end{array}\right\} \\
\leq \psi\left(\max \left\{\begin{array}{l}
p(T x, T y), p(T y, T z), \\
p(T z, T w), p(T w, T x)
\end{array}\right\}\right) \\
-\phi\left(\max \left\{\begin{array}{l}
p(T x, T y), p(T y, T z), \\
p(T z, T w), p(T w, T x)
\end{array}\right\}\right),
\end{gathered}
$$

Hence

$$
\max \left\{\begin{array}{l}
p(T x, T y), p(T y, T z) \\
p(T z, T w), p(T w, T x)
\end{array}\right\}=0
$$

since $\phi(t)>0$ for $t>0$.

$\therefore T x=T y, T y=T z, T z=T w, T w=T x$.

Since $T$ is injuctive, we have $x=y=z=w$.

Thus $F$ has a quadruple fixed point of the form $(x, x, x, x)$.

The following example illustrates our Theorem 2.2 
Example 2.1. Let $X=[0,1], p(x, y)=\max \{x, y\}$ and $T: X \rightarrow X$ be defined by $T(x)=\frac{x}{2}$. Let $F: X \times X \times X \times X \rightarrow X$ by $F(x, y, z, w)=$ $\frac{x^{2}+y^{2}+z^{2}+w^{2}}{8}$ and $\psi, \phi:[0, \infty) \rightarrow[0, \infty)$ by $\psi(t)=t, \phi(t)=\frac{t}{2}$ clearly all conditions of Theorem 2.2 are satisfied and

$$
\begin{aligned}
& \psi(p(T F(x, y, z, w), T F(u, v, r, t)))=p\left(\frac{F(x, y, z, w)}{2}, \frac{F(u, v, r, t)}{2}\right) \\
= & \max \left\{\frac{x^{2}+y^{2}+z^{2}+w^{2}}{16}, \frac{u^{2}+v^{2}+r^{2}+t^{2}}{16}\right\} \\
= & \frac{1}{16} \max \left\{x^{2}+y^{2}+z^{2}+w^{2}, u^{2}+v^{2}+r^{2}+t^{2}\right\} \\
= & \frac{1}{16}\left[\max \left\{x^{2}, u^{2}\right\}+\max \left\{y^{2}, v^{2}\right\}+\max \left\{z^{2}, r^{2}\right\}+\max \left\{w^{2}, t^{2}\right\}\right] \\
\leq & \frac{1}{4} \max \left\{\max \left\{x^{2}, u^{2}\right\}, \max \left\{y^{2}, v^{2}\right\}, \max \left\{z^{2}, r^{2}\right\}, \max \left\{w^{2}, t^{2}\right\}\right\} \\
\leq & \frac{1}{4} \max \{\max \{x, u\}, \max \{y, v\}, \max \{z, r\}, \max \{w, t\}\} \\
\leq & \frac{1}{2} \max \{\max \{T x, T u\}, \max \{T y, T v\}, \max \{T z, T r\}, \max \{T w, T t\}\} \\
= & \psi(\max \{p(T x, T u), p(T y, T v), p(T z, T r), p(T w, T t)\}) \\
& \quad-\phi(\max \{p(T x, T u), p(T y, T v), p(T z, T r), p(T w, T t)\}) .
\end{aligned}
$$

Clearly $(0,0,0,0)$ is quadruple fixed point of $F$.

\section{Application}

In this section, we study the existence of a unique solution to an initial value problem, as an application to Theorem 2.2.

Consider the initial value problem

$$
x^{1}\left(t_{1}\right)=l\left(t_{1}, x\left(t_{1}\right), x\left(t_{1}\right), x\left(t_{1}\right), x\left(t_{1}\right)\right), t_{1} \in I=[0,1], x(0)=x_{0}
$$

where $l: I \times\left[\frac{x_{0}}{8}, \infty\right) \times\left[\frac{x_{0}}{8}, \infty\right) \times\left[\frac{x_{0}}{8}, \infty\right) \times\left[\frac{x_{0}}{8}, \infty\right) \rightarrow\left[\frac{x_{0}}{8}, \infty\right)$ and $x_{0} \in \mathbb{R}^{+}$.

Theorem 3.1. Consider the initial value problem 18 with

$$
l \in C\left(I \times\left[\frac{x_{0}}{8}, \infty\right) \times\left[\frac{x_{0}}{8}, \infty\right) \times\left[\frac{x_{0}}{8}, \infty\right) \times\left[\frac{x_{0}}{8}, \infty\right)\right)
$$


and

$$
\begin{aligned}
& \int_{0}^{t_{1}} l(s, x(s), y(s), z(s), w(s)) \mathrm{d} s \leq \\
& \leq \max \left\{\begin{array}{l}
\frac{1}{2} \int_{0}^{t_{1}} l(s, x(s), x(s), x(s), x(s)) \mathrm{d} s-\frac{x_{0}}{4}, \\
\frac{1}{2} \int_{0}^{t_{1}} l(s, y(s), y(s), y(s), y(s)) \mathrm{d} s-\frac{x_{0}}{4}, \\
\frac{1}{2} \int_{0}^{t_{1}} l(s, z(s), z(s), z(s), z(s)) \mathrm{d} s-\frac{x_{0}}{4}, \\
\frac{1}{2} \int_{0}^{t_{1}} l(s, w(s), w(s), w(s), w(s)) \mathrm{d} s-\frac{x_{0}}{4}
\end{array}\right\} .
\end{aligned}
$$

Then there exists unique solution in $C\left(I,\left[\frac{x_{0}}{8}, \infty\right)\right)$ for the initial value problem 18.

Proof. The integral equation corresponding to initial value problem 18 is

$$
x(t)=x_{0}+\int_{0}^{t} l(s, x(s), x(s), x(s), x(s)) \mathrm{d} s .
$$

Let $X=C\left(I,\left[\frac{x_{0}}{8}, \infty\right)\right)$ and $p(x, y)=\max \left\{x-\frac{x_{0}}{8}, y-\frac{x_{0}}{8}\right\}$ for $x, y \in X$. Define ICS mapping $T: X \rightarrow X$ by $T x=\frac{x}{4}, \psi, \phi:[0, \infty) \rightarrow[0, \infty)$ by $\psi(t)=t, \phi(t)=\frac{t}{2}$ and $F: X \times X \times X \rightarrow X$ by

$$
F(x, y, z, w)\left(t_{1}\right)=x_{0}+\int_{0}^{t_{1}} l(s, x(s), y(s), z(s), w(s)) \mathrm{d} s .
$$

Now

$$
\begin{aligned}
& \psi\left(p\left(T F(x, y, z, w)\left(t_{1}\right), F(u, v, r, t)\left(t_{1}\right)\right)\right) \\
= & \max \left\{\frac{F(x, y, z, w)}{4}-\frac{x_{0}}{8}, \frac{F(u, v, r, t)}{4}-\frac{x_{0}}{8}\right\} \\
= & \frac{1}{4} \max \left\{\begin{array}{c}
\frac{x_{0}}{2}+\int_{0}^{t_{1}} l(s, x(s), y(s), z(s), w(s)) \mathrm{d} s, \\
\frac{x_{0}}{2}+\int_{0}^{t_{1}} l(s, u(s), v(s), r(s), t(s)) \mathrm{d} s
\end{array}\right\}
\end{aligned}
$$




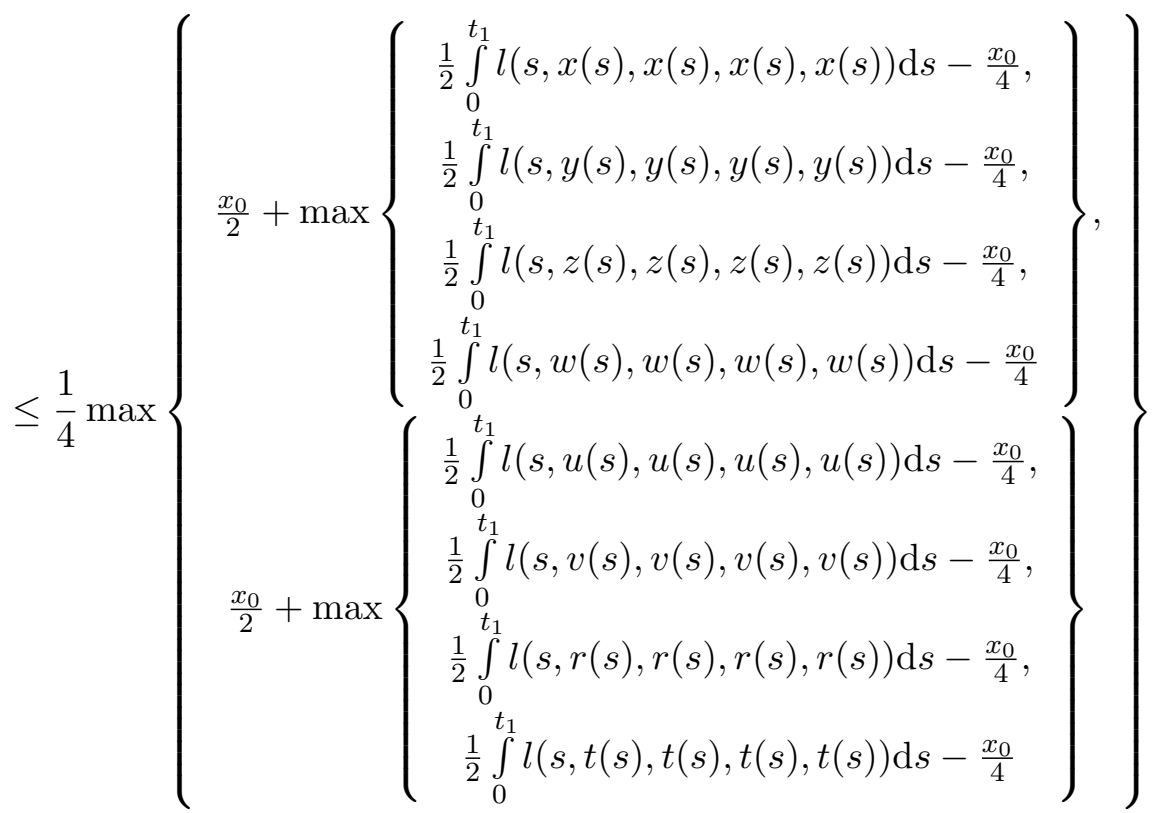

$$
\begin{aligned}
& =\frac{1}{2} \max \left\{\begin{array}{c}
\max \left\{\frac{x\left(t_{1}\right)}{4}-\frac{x_{0}}{8}, \frac{y\left(t_{1}\right)}{4}-\frac{x_{0}}{8}, \frac{z\left(t_{1}\right)}{4}-\frac{x_{0}}{8}, \frac{w\left(t_{1}\right)}{4}-\frac{x_{0}}{8}\right\}, \\
\max \left\{\frac{u\left(t_{1}\right)}{4}-\frac{x_{0}}{8}, \frac{v\left(t_{1}\right)}{4}-\frac{x_{0}}{8}, \frac{r\left(t_{1}\right)}{4}-\frac{x_{0}}{8}, \frac{t\left(t_{1}\right)}{4}-\frac{x_{0}}{8}\right\}
\end{array}\right\} \\
& =\frac{1}{2} \max \left\{\begin{array}{c}
\max \left\{T x\left(t_{1}\right)-\frac{x_{0}}{8}, T u\left(t_{1}\right)-\frac{x_{0}}{8}\right\}, \\
\max \left\{T y\left(t_{1}\right)-\frac{x_{0}}{8}, T v\left(t_{1}\right)-\frac{x_{0}}{8}\right\}, \\
\max \left\{T z\left(t_{1}\right)-\frac{x_{0}}{8}, T r\left(t_{1}\right)-\frac{x_{0}}{8}\right\}, \\
\max \left\{T w\left(t_{1}\right)-\frac{x_{0}}{8}, T t\left(t_{1}\right)-\frac{x_{0}}{8}\right\}
\end{array}\right\} \\
& =\frac{1}{2} \max \{p(T x, T u), p(T y, T v), p(T z, T r), p(T w, T t)\} \\
& =\psi\left(\max \left\{\begin{array}{c}
p(T x, T u), p(T y, T v), \\
p(T z, T r), p(T w, T t)
\end{array}\right\}\right) \\
& -\phi\left(\max \left\{\begin{array}{c}
p(T x, T u), p(T y, T v), \\
p(T z, T r), p(T w, T t)
\end{array}\right\}\right)
\end{aligned}
$$

Thus $F$ satisfies the condition (2.2.1) of Theorem 2.2. From Theorem 2.2, we conclude that $F$ has a quadruple fixed point. In particular $x(t)$ is the unique solution of the integral equation (19).

\section{REFERENCES}

[1] T. Abdeljawad, E. Karapınar, K. Tas,Existence and uniqueness of a common fixed point on partial metric spaces, Appl. Math. Lett. 24(11) (2011), 1894-1899.

[2] I. Altun, F. Sola and H. Simsek, Generalized contractions on partial metric spaces, Topology and its Applications, 157(18) (2010), 2778-2785. 
[3] K.P. Chi, On a fixed point theorem for certain class of maps satisfying a contractive condition depended on an another function, Lobachevskii J. Math., 30(4) (2009), 289-291.

[4] E. Karapinar, I.M. Erhan, Fixed point theorems for operators on partial metric spaces, Applied Mathematics Letters 24(11) (2011), 1900-1904, 10.1016/j.aml.2011.05.013.

[5] E. Karapínar, Quadruple fixed point theorems for weak $\phi$ - contractions, ISRN Mathematical Analysis, Article ID 989423 (2011), 15 pages.

[6] E. Karapínar and V. Berinde, Quadruple fixed point theorems for nonlinear contractions in partially ordered metric spaces, Banach Journal of Mathematical Analysis, 6(1) (2012), 74-89.

[7] N.V. Luong, N.X. Thuan and T.T. Hai, Coupled fixed point theorems in partially ordered metric spaces depended on an another function, Bulletin of mathematical analysis and applications, 3(3) (2011), 129-140.

[8] S.G. Matthews. Partial metric topology, Research Report 212, Dept. of Computer Science, University of Warwick, 1992.

[9] S.G. Matthews, Partial metric topology, in Proceedings of the 8th Summer Conference on General Topology and Applications, Annals of the New York Academy of Sciences, 728 (1994), 183-197.

\author{
K.P.R. RAO \\ Department of Mathematics \\ Acharya Nagarjuna University \\ Nagarjuna Nagar, Guntur - 522510 \\ ANDHRA PRADESH \\ INDIA \\ E-mail address: kprrao2004@yahoo.com
}

\section{G.N.V. KISHORE}

Department of Mathematics

KL UNIVERSITY

VAddeswaram, Guntur - 522502

ANDHRA Pradesh

INDIA

E-mail address: kishore.apr2@gmail.com

gnvkishore@kluniversity.in

\section{K.V. Siva Parvathi}

Department of Applied Mathematics

Krishna University - M.R. Appa Row P.G.Center

Nuzvid-521 201, Andhra Pradesh

INDIA

E-mail address: kvsp1979@yahoo.com 